\title{
SENSORY TEXTURE OF DATE FRUITS AS A FUNCTION OF PHYSICOCHEMICAL PROPERTIES AND ITS USE IN DATE CLASSIFICATION
}

\author{
V. Singh ${ }^{a *}$, N. Guizania ${ }^{a}$, I. Al-Zakwani ${ }^{a}$, Q. Al-Shamsi ${ }^{b}$, A. Al-Alawi a and M.S. Rahman ${ }^{a}$ \\ ${ }^{a}$ Department of Food Science and Nutrition, College of Agricultural and Marine Sciences, \\ Sultan Qaboos University, P. O. Box 34, Al-Khod 123, Muscat, Oman \\ ${ }^{\mathrm{b}}$ Dubai Municipality, P.O. Box 67, Dubai. UAE
}

(Received: 19 September 2013; accepted: 20 December 2013)

\begin{abstract}
Eight descriptive sensory textural attributes of whole date fruit were evaluated by twenty trained panel members and correlated with sixteen physicochemical properties. All sensory parameters, except gumminess, significantly correlated $(\mathrm{P}<0.05)$ with pectin, crude fibre, and moisture content. In addition, sensory hardness, cohesiveness, elasticity, and resilience correlated significantly with length of whole fruit $(\mathrm{P}<0.05)$, sensory adhesiveness with glucose content $(\mathrm{P}<0.05)$, sensory chewiness with mass of whole fruit $(\mathrm{P}<0.05)$, and sensory gumminess with fructose, glucose, and total sugar content $(\mathrm{P}<0.10)$. Sweetness, however, correlated only with moisture content $(\mathrm{P}<0.05)$. CA and the biplot (i.e. including all products, their sensory texture and physicochemical attributes) generated through PCA recognized three groups of dates as hard-chewy, soft-(medium-chewy), and soft-(nonadhesive).
\end{abstract}

Keywords: date, moisture, sensory, PCA, classification

Date palm fruit is a berry consisting of a pericarp and a pit. The pericarp constitutes 85-90\% of the total fruit mass and it is composed of exocarp, fleshy mesocarp, and papery endocarp (Hussein et al., 1998). Dates can be consumed as fresh fruit at khalal and rutab stages (immature stages with short shelf life) or at tamar stage (mature stage with good storability). It is important to know the physicochemical, mechanical, structural, textural, and sensory properties of dates for their processing, storage stability, and consumer acceptability. The rank of the most preferred sensory quality attributes of dates at tamar stage of maturity were high for colour, appearance, sweetness, medium for fruit size, chewiness, solubility, flesh thickness, and low for elasticity, mouth feel, and shear force (IsmaIL et al., 2001).

Sensory properties of a food are the ultimate considerations used by the consumers for accepting a product. Sensory properties are mainly measured by expert-panels, trained panels, and consumer panels. Earlier sensory profile of 7 cultivars of date fruit from Tunisia showed a great morphological and physicochemical diversity among the tested cultivars (BEN IsmaIL et al., 2013). The sensory attributes of date syrup (AвBEs et al., 2011) and date jams (BEsBes et al., 2009) were also determined. Studying the effect of storage conditions on the sensory profile of two cultivars of date fruit, Ismall and co-workers (2008) observed behavioural variation in measured physicochemical and sensory attributes between the two cultivars under matching storage conditions. They concluded that most of the variation of the stored dates comes from "size", followed by "texture" variables. In the literature, very

\footnotetext{
* To whom correspondence should be addressed.

Phone: +968 2414 1256; e-mail: vandita31@gmail.com; guizani@sqe.edu.om
} 
limited studies have assessed the relationships between the sensory texture and physicochemical properties of date fruit. The overall objective of this study was to determine the sensory texture of nine batches of dates with different quality levels and to determine their relationship with 16 physicochemical properties.

\section{Materials and methods}

\subsection{Descriptive sensory analysis}

Fifteen physicochemical properties of nine batches of dates were measured previously (SiNGH et al., 2013). Total polyphenols was measured by the method of SINGH and co-workers (2012). Sensory texture analysis was conducted with a panel consisting of a panel leader and twenty panel members.

\subsection{Panel members}

The panel consisted of twenty members (10 males and 10 females) within the age of 21-25 (students from the CAMS, Sultan Qaboos University). Most of the panel members consume dates regularly (i.e. every day) and none of them had teeth problems or diabetes, and all were non-smokers. The members agreed to participate in the training (three sessions, $2 \mathrm{~h}$ each) and to perform the sensory texture analysis, i.e. two sessions a day with at least 1 hour interval for one attribute. However, sweetness and hardness were measured at the same time.

\subsection{Sample preparation}

Different batches of dates were removed from the freezer and placed in the sensory preparation room for at least $2 \mathrm{~h}$ prior to analysis. Dates were then randomly selected from different polyethylene bags and placed on a disposable plate marked with 3 digit coding.

\subsection{Training}

The panel leader organized three training sessions before assessing the samples. In the first training session, panel members were trained on the concept of sensory properties including definition of different textural attributes. In the second session, they were trained on the scale and standard used for different attributes. In the third session, they were trained to assess the textural attributes considering its definition, following the procedure and standards as included in Table 1.

\subsection{Sensory evaluation}

The panel members received different date samples in the sensory evaluation booth. Panel members were instructed to remove the seed and place the date flesh in between the molar teeth and assess the sensory attributes based on the definitions and standards they received. They were then asked to include their rating on a scale considering the procedures provided in Table 1. Water was used to wash the mouth twice in between assessments. All sessions were conducted in a climate-controlled sensory analysis booth (i.e. $20^{\circ} \mathrm{C}$ room temperature, positive air pressure) and under white fluorescent lighting. Detailed statistical analysis procedures were presented in an earlier paper by SINGH and co-workers (2013). 
SINGH et al.: SENSORY TEXTURE OF DATE FRUITS

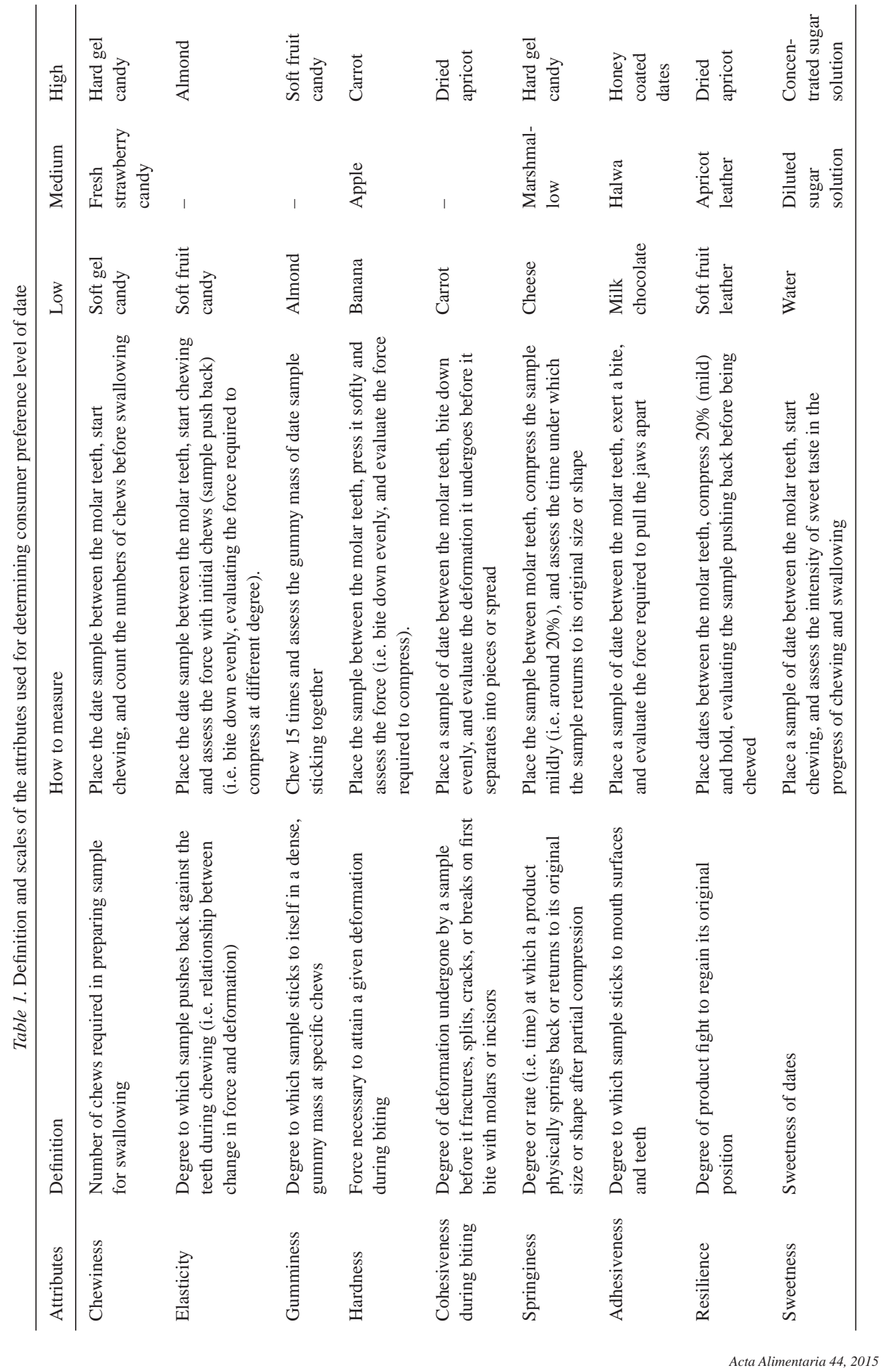




\section{Results and discussion}

\subsection{Correlation between sensory textural attributes and physicochemical properties}

Table 2 presents the sensory attributes of 9 batches of dates as a function of moisture content. Pearson's correlation showed that sensory hardness (HAS) was negatively correlated with moisture content (XW), crude fibre (CF); and positively correlated with length of whole date fruit (LT), pectin $(\mathrm{PE})(\mathrm{P}<0.05)$, and sugar index $(\mathrm{SI})(\mathrm{P}<0.10)$. Spearman's correlations did not improve the relation between the HAS and physicochemical properties $(\mathrm{P}<0.05$ or $\mathrm{P}<0.1)$. Similarly, hardness of cheese decreased linearly with moisture and increased with increasing starch content (NoRONHA et al., 2007), while it was positively correlated with pectin content in pear (Ying et al., 2011) and apple (BILly et al., 2008).

Table 2. Sensory analysis as a function of moisture content of date samples

\begin{tabular}{lcccccccccc}
\hline CP $^{\mathrm{a}}$ & XW & HAS & ADS & SPS & COS & RES & GUS & CHS & ES & SW \\
\hline A & $17.9(0.1)$ & $3.3(1.3)$ & $4.8(2)$ & $3.9(1.7)$ & $4.7(1.7)$ & $4.1(2.0)$ & $5.9(1.7)$ & $24(13.4)$ & $3.8(1.4)$ & $5.9(1.5)$ \\
B & $20.6(0.3)$ & $2.8(1.7)$ & $4.2(1.5)$ & $3.0(1.4)$ & $4.0(2.2)$ & $3.3(2.0)$ & $5.3(2.1)$ & $17(10.6)$ & $2.5(1.6)$ & $5.9(1.3)$ \\
C & $17.9(0.2)$ & $4.5(2.1)$ & $4.5(2.1)$ & $3.5(1.4)$ & $4.9(1.5)$ & $4.3(1.7)$ & $5.4(1.7)$ & $20(13.5)$ & $4.2(1.4)$ & $5.3(1.9)$ \\
D & $16.1(0.3)$ & $5.8(2.5)$ & $4.6(2.2)$ & $5.2(2.3)$ & $5.0(2.2)$ & $4.9(2.2)$ & $4.5(2.2)$ & $23(10.8)$ & $5.8(1.7)$ & $5.1(2.0)$ \\
E & $22.4(0.6)$ & $3.3(1.4)$ & $4.4(1.6)$ & $3.5(2.0)$ & $4.3(2.0)$ & $3.6(1.9)$ & $5.3(2.1)$ & $20(12.3)$ & $3.4(1.2)$ & $6.7(1.8)$ \\
F & $19.5(0.2)$ & $4.1(1.8)$ & $3.7(1.4)$ & $3.4(2.0)$ & $4.1(1.0)$ & $3.7(1.9)$ & $3.9(1.7)$ & $20(10.5)$ & $3.6(2.0)$ & $6.1(1.5)$ \\
G & $17.7(0.3)$ & $3.7(1.5)$ & $4.8(2.0)$ & $4.3(1.7)$ & $4.5(1.6)$ & $3.6(1.5)$ & $4.5(1.9)$ & $21(11.7)$ & $4.1(1.1)$ & $5.6(1.7)$ \\
H & $16.1(0.1)$ & $6.2(1.4)$ & $5.4(2.4)$ & $4.4(1.7)$ & $5.8(1.5)$ & $4.6(1.7)$ & $5.2(2.0)$ & $27(16.3)$ & $6.2(1.7)$ & $5.9(1.8)$ \\
I & $16.5(0.2)$ & $6.8(1.7)$ & $4.9(2.1)$ & $4.2(1.7)$ & $6.4(1.6)$ & $5.1(2.1)$ & $5.7(2.2)$ & $32(18.3)$ & $6.2(1.9)$ & $5.6(1.1)$ \\
DT & $\mathrm{P}<0.001$ & $\mathrm{P}<0.001$ & $\mathrm{P}<0.001$ & $\mathrm{P}<0.001$ & $\mathrm{P}<0.001$ & $\mathrm{P}<0.001$ & $\mathrm{P}<0.001$ & $\mathrm{P}<0.001$ & $\mathrm{P}<0.001$ & $\mathrm{P}<0.001$ \\
\hline
\end{tabular}

$\mathrm{CP}^{\mathrm{a}}$ : commercial products; XW: moisture content; HAS: sensory hardness; ADS: sensory adhesiveness; SPS: sensory springiness; COS: sensory cohesiveness; RES: sensory resilience; GUS: sensory gumminess; CHS: sensory chewiness; ES: sensory elasticity; SW: sensory sweetness; DT: discrepancy test for each column

Pearson's correlation showed that sensory adhesiveness (ADS) was positively correlated with glucose $(\mathrm{GL})$, SI $(\mathrm{P}<0.05)$, total sugar $(\mathrm{TS})$, and $\mathrm{PE}(\mathrm{P}<0.1)$; and negatively correlated with XW $(\mathrm{P}<0.1)$. Spearman's correlations did not improve the relation for GL $(\mathrm{P}<0.05$ or $\mathrm{P}<0.1)$, but improved negative correlations for $\mathrm{CF}$ and colour a value $(\mathrm{P}<0.05)$. Sensory springiness (SPS) was correlated negatively with XW $(\mathrm{P}<0.05)$ and $\mathrm{CF}(\mathrm{P}<0.1)$ and positively with SI $(\mathrm{P}<0.05)$ and $\mathrm{PE}(\mathrm{P}<0.10)$. Spearman's correlation was improved for $\mathrm{CF}(\mathrm{P}<0.05)$, but not for other attributes.

Pearson's correlation was positive between sensory cohesiveness (COS) and LT, PE, and SI $(\mathrm{P}<0.05)$, and mass of whole date fruit $(\mathrm{MA})(\mathrm{P}<0.1)$ and negative with $\mathrm{XW}$ and $\mathrm{CF}$ $(\mathrm{P}<0.05)$. Spearman's correlations did not improve the relation $(\mathrm{P}>0.1)$. Sensory resilience (RES) was correlated positively with LT and $\mathrm{PE}(\mathrm{P}<0.05)$, and MA $(\mathrm{P}<0.1)$ and negatively correlated with XW $(\mathrm{P}<0.05)$ and $\mathrm{CF}(\mathrm{P}<0.1)$. In this case, Spearman's correlations did not improve for RES ( $>>0.1)$. Sensory gumminess (GUS) was positively correlated (Pearson's) with fructose (FR), GL, and TS $(\mathrm{P}<0.1)$. 
Spearman's correlations showed poor correlations ( $\mathrm{P}>0.1)$ as compared to the Pearson's correlations. Sensory chewiness was correlated positively with MA, LT, and $\mathrm{PE}(\mathrm{P}<0.05)$ and negatively correlated with XW and CF $(\mathrm{P}<0.05)$. Spearman's correlation improved positive correlation of SI $(\mathrm{P}<0.1)$, and decreased correlation for MA $(\mathrm{P}<0.1)$. Sensory elasticity (ES) was positively correlated with LT, PE, and SI and negatively with XW and CF $(\mathrm{P}<0.05)$. Sensory sweetness (SW) was correlated with XW $(\mathrm{P}<0.05)$ and SI $(\mathrm{P}<0.1)$. Spearman's test improved the correlation for sucrose content (SU) in dates $(\mathrm{P}<0.1)$. It is interesting that most of the sensory textural attributes correlated with XW, PE, and CF.

All sensory textural attributes of date fruit (except gumminess) negatively correlated with moisture and crude fibre; while it was positively correlated with the pectin content. Sugar content positively correlated with all sensory attributes except for cohesiveness and elasticity. Water is a well-known plasticizer that is able to rupture hydrogen bonds in the solid matrix, thus softer texture is formed with increasing water content. Higher sugar and pectin produced harder texture due to their thickening or gelling effect as also pointed out by BESBES and co-workers (2009) in the case of date jams. However, crude fibre influenced the gelling structure of sugar and pectin by damaging or weakening the firm gels.

\subsection{Cluster Analysis (CA) and Principal Component Analysis (PCA)}

A CA of dates based on Ward's method revealed 3 groups (Fig. 1). The PCA analysis of the same groups identified by CA showed six principal components (95.1\% total variance) with Eigen values close to 1 . These PCs explained 38.3, 23.7, 12.7, 12.6, 4.6, and 3.3\% of the total variance, respectively. Figure 2 presents the bi-plot including all date fruit sensory texture and physicochemical properties. The first axis correlated well with MA, LT, HAS, RES, and $\mathrm{CF}$, and corresponds to hardness (i.e. force required to compress or deform). The second axis was strongly correlated to colour-b value, PE, XW, SPS, CHS, COS, and RES, and corresponds to structure regain ability after deformation. The third axis was strongly correlated with colour-L value, SI, and SW, and corresponds to the sweetness. The fourth axis was strongly correlated with ADS, GUS, and SW, and corresponds to the surface sticking ability. The fifth axis was strongly correlated with SU and GL, and corresponds to low intensity sweeteners. The sixth axis was strongly correlated with SU, FR, TS, pH, TP, and width of whole fruits (WD) and corresponds to $\mathrm{pH}$.

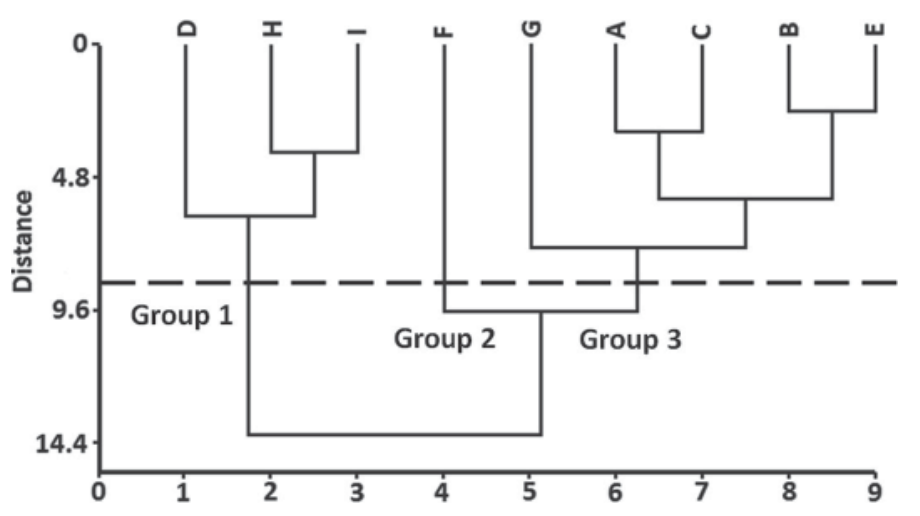

Fig. 1. Dendrogram of the cluster analysis for different date samples [Group 1 (D, H, I); Group 2 (F); Group 3 (G, A, C, B, E)] 
This shows that complete sensory texture assessment can be explained by six major factors, among them three evolved from structure (force-deformation, structure regain ability, and surface sticking ability), and two from the composition (sugars and $\mathrm{pH}$ ). Similar results were observed in the cases of sensory texture and physicochemical properties of 12 types of cheese (full-fat, low-fat, and reduced-fat) when analyzed by PCA (LTEIF et al., 2009). HAS and CHS belonged to the first axis, while SPS and COS belonged to the second axis. However, in the case of cheese, springiness was opposite to cohesiveness. ADS belonged to the fourth axis, while taste (i.e. saltiness) belonged to the third axis, which was similar to the sweetness of dates. In the case of cooked chicken breast, it was observed that the measured HAS, CHS, and SPS showed similarity to our results; however, other textural attributes were not considered in the study by Liu and co-workers, (2004).

The products' sensory textural attributes and physicochemical properties were plotted considering PC 2 and PC 1 (Fig. 2). The Group 1 (D, H, I) on the right hand side of the PCA plot includes hard and chewy (i.e. hard-chewy) dates. On the opposite side of Group 1, the dates in Group 3 (G, A, C, B, E) are high in water, soft, low-chewy, and medium-gummy [i.e. soft-(medium-gummy)]. Similarly, dates in Group 2 (F) are high in water, soft, and nonadhesive [i.e. soft-(non-adhesive)]. Group 2 is medium-chewy, while Group 3 is nonadhesive. In addition, Group 1 is low sweet, while Group 2 and Group 3 are sweeter. This indicated that different brands of date fruits with different degrees of chewiness and gumminess could be branded based on the desired consumer preferences.

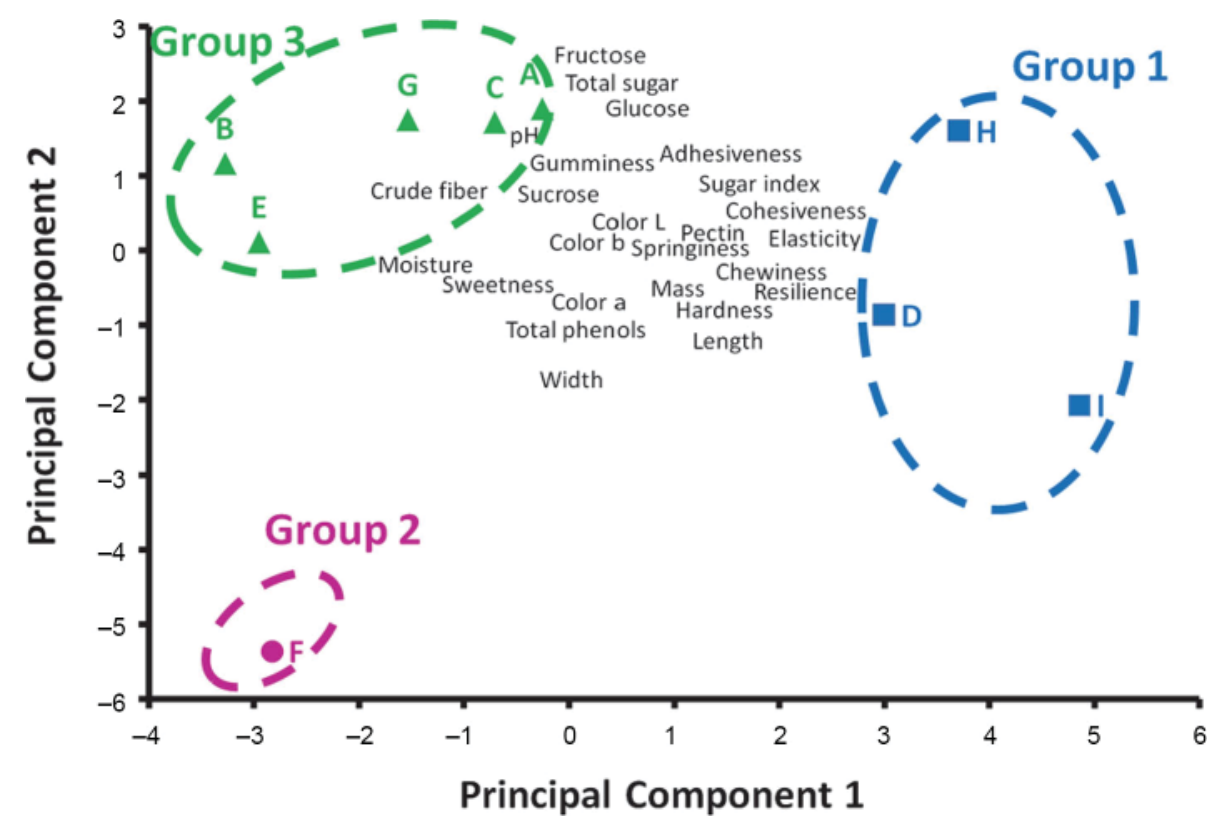

Fig. 2. Bi-plot of Principal Component 2 and Principal Component 1 


\section{Conclusions}

With the exception of gumminess, all sensory texture of date fruit negatively correlated with moisture and crude fibre; while it was positively correlated with the pectin content. Sugar content negatively correlated with all sensory attributes with the exception of cohesiveness and elasticity. The results could be used to explore how physicochemical properties contributed to the sensory texture and could help to select and process the fruit with their desired sensory attributes. The CA and PCA suggest sorting dates into three groups as hardchewy, soft-(medium-chewy), and soft-(non-adhesive). This classification could be useful for the pricing of dates in the local and international markets based on their desired attributes.

The participation of the tasting panel, technical help by Ms. Aditi ChatTERJEe and Ms. Abeer Al-Aamri and financial assistance provided by Sultan Qaboos University are greatly acknowledged.

\section{References}

Abbes, F., Bounziz, M.A., Blecker, C., Masmoudi, M., Attia, H. \& Souhail, B. (2011): Date syrup: Effect of hydrolytic enzymes (pectinase/cellulase) on physicochemical characteristics, sensory and functional properties. LWT - Food Sci. Technol., 44, 1827-1834.

Ben Ismail, H., Duendoubi, N., Kodia, A., Ben Hassine, D. \& Ben Slama, M. (2013): Physicochemical characterization and sensory profile of 7 principal Tunisian date cultivars. Emir. J. Food Agric., 25, 331-341.

Besbes, S., Drira, L., Blecker, C., Deroanne, C. \& Attia, H. (2009): Adding value to hard date (Phoenix dactylifera L.): Compositional, functional and sensory characteristics of date jam. Food Chem., 112, 406-411.

Billy, L., Mehinagic, E., Royer, G., Renard, C.M.G.C., Arvisenet, G., Prost, C. \& Jourjon, F. (2008): Relationship between texture and pectin composition of two apple cultivars during storage. Postharvest Biol. Technol., 47, 315-324.

Hussein, A.S., Alhadrami, G.A. \& Khalil, Y.H. (1998): The use of dates and date pits in broiler starter and finisher diets. Bioresource Technol., 66, 219-223.

Ismail, B., Haffar, I., BaAlbaki, R. \& Henry, J. (2001): Development of a total quality scoring system based on consumer preference weightings and sensory profiles: application to fruit dates (Tamr). Food Qual. Prefer., 12, 499-506.

Ismail, B., Haffar, I., BaAlbaki, R. \& Henry, J. ( 2008). Physicochemical characteristics and sensory quality of two date varieties under commercial and industrial storage conditions. LWT - Food Sci. Technol., 41, 896-904.

Liu, Y., Lyon, B., Windham, W., Lyon, C. \& Savage, E. (2004): Principal component analysis of physical, color, and sensory characteristics of chicken breasts deboned at two, four, six, and twenty-four hours postmortem. Poultry Sci., 83, 101-108.

Lteif, L., Olabi, A., Kebbe Baghdadi, O. \& Toufeili, I. (2009): The characterization of the physicochemical and sensory properties of full-fat, reduced-fat, and low-fat ovine and bovine Halloumi. J. Dairy Sci., 92, 41354145 .

Noronha, N., O’Riordan, E.D. \& O’Sullivan, M. (2007): Replacement of fat with functional fibre in imitation cheese. Int. Dairy J., 17, 1073-1082.

Singh, V., Guizani, N., Essa, M.M., HaKkim, F.L. \& Rahman, M.S. (2012): Comparative analysis of total phenolics, flavonoid content and antioxidant profile of different date varieties (Phoenix dactylifera L.) from Sultanate of Oman. Int. Food Res. J., 19, 1063-1070.

Singh, V., Guizani, N., Al-Alawi, A., Claereboudt, M. \& Rahman, M.S. (2013): Instrumental texture profile analysis (TPA) of date fruits as a function of its physicochemical properties. Ind. Crop. Prod., 50, 866-873.

Ying, Y., XIAO-YAN, L.U., YAn, L., Yu-QIONG, Y., Bing-GANG, M.A. \& LeI, W. (2011): Study on correlation between firmness and pectin content of different pear cultivars; Northern Horticulture, Available at:http://en.cnki.com. cn/Article_en/CJFDTotal-BFYY201115005.htm (last accessed: 1 December 2013) 